\title{
Verspieltes Kapital? Der gesellschaftliche Rollenwandel des Samuraistandes im Zuge der Meiji-Restauration
}

\author{
Maximilian Gröber \\ Kerngebiet: Neuzeit \\ eingereicht bei: assoz. Prof. Dr. Elena Taddei \\ eingereicht im: SoSe 2020 \\ Rubrik: Bachelor-Arbeit
}

\begin{abstract}
The change in the social role of the Samurai ranks during the Meiji Restoration
\end{abstract}

The creation of the nation state and its influence on the respective societies characterized the 19th century - not only in Europe. As a result of the Meiji Restoration, which initiated the formation of modern Japan in the 1860s, feudalism came to an end. By using Pierre Bourdieu's theory of capital and class distinction, the goal of this study is to illuminate and evaluate the social status of the former warrior nobility, the samurai, under these changed circumstances.

\section{Einleitung}

„Kurz, man hüte sich, zu notwendigen und intrinsischen Merkmalen irgendeiner sozialen Gruppe (Adel, Samurais, aber auch Arbeiter oder Angestellte) zu erklären, was in Wirklichkeit Merkmale sind, die ihr zu einem bestimmten Zeitpunkt aufgrund ihrer Position in einem bestimmten sozialen Raum und bei einem bestimmten Stand des Angebots an möglichen Praktiken und Gütern zukommen."1

Pierre Bourdieu, Sozialer Raum, symbolischer Raum (1989), in: Susanne Hauser/Christa Kamleithner/Roland Meyer (Hrsg.), Architekturwissen. Grundlagentexte aus den Kulturwissenschaften, Bd. 1, Bielefeld 2011, S. 304-313, hier S. 306. 
Gerade die Gruppe der Samurai, auf welche Bourdieu in diesem Zitat am Rande eingeht, erfuhr mit dem Ende der ständischen Ordnung in der zweiten Hälfte des 19. Jahrhunderts einen einschneidenden Wandel ihrer Rolle innerhalb der japanischen Gesellschaft. Oberflächlich betrachtet scheint die ruckartige Auflösung eines Feudalsystems, wie sie sich in Japan Ende der 1860er-Jahre zutrug, den Adel - in diesem Falle die Samurai - zwangsläufig in eine sozial schlechtere Position zu zwingen. Der mehrheitlich geringfügige Widerstand der Kriegeraristokratie gegen diese Umbrüche deutet allerdings auf andere Entwicklungen hin.

Ziel der vorliegenden Arbeit ist es daher, durch die genauere Beleuchtung dieser Prozesse die soziale Platzierung der Mitglieder des Samuraistandes² im modernen japanischen Nationalstaat nachvollziehen zu können. Methodisch sollen dabei die Theorien Pierre Bourdieus auf das Beispiel der Samurai angewandt werden, um deren ökonomisches, kulturelles und soziales Kapital - also ihre „wirklichen Merkmale” - zu identifizieren. Die Untersuchung folgt dabei der Annahme, dass es zu keinen gravierenden Verlusten im Bereich dieser Kapitalarten kam und der Stand der Samurai demnach keinem sozialen Abstieg ausgesetzt war.

Zur Einführung werden die soziologischen Überlegungen Bourdieus kurz dargelegt und in einem nächsten Schritt die japanische Gesellschaft der frühen Neuzeit samt ihrer zentralen Akteur*innen skizziert. Besonderen Raum nimmt dabei die Beschreibung des Samuraistandes ein. Die Ereignisse der sogenannten Meiji-Restauration, welche zum Ende des Feudalismus in Japan und zur Herrschaftsübernahme des Kaisers führten, stehen im darauffolgenden Abschnitt im Zentrum. Abschließend wird die gesellschaftliche Stellung des ehemaligen Kriegeradels unter diesen veränderten Bedingungen beleuchtet und bewertet.

Betreffend der für diese Arbeit herangezogenen Literatur ist zu sagen, dass es im deutschen Sprachraum eine rege Auseinandersetzung mit der japanischen Geschichte gibt. Als grundlegende Standardwerke wären hier vor allem die Monografien von Reinhard Zöllner ${ }^{3}$ sowie Gerhard Krebs ${ }^{4}$ zu nennen. Mit dem von Josef Kreiner herausgegebenen Sammelwerk ${ }^{5}$ findet sich außerdem eine sehr aktuelle und prägnante Zusammenfassung der gesamten Geschichte Japans. Zur Behandlung der aufgeworfenen Fragestellungen bezüglich der sozialen Stellung der Samurai und ihrer Kapitalarten bildet vor allem der ausführliche Aufsatz von Wolfgang Schwentker, in welchem er die bedeutendsten Forschungsergebnisse der vergangenen Jahrzehnte nochmals

2 Wenn im Folgenden von den Privilegien des Samuraistandes die Rede ist, bezieht sich dies ausschließlich auf dessen männliche Vertreter. Hierfür sind vor allem zwei Gründe verantwortlich: Zum einen ergeben sich aus den vorherrschenden Geschlechtervorstellungen einer gewissen Zeit verschiedene Rollenzuschreibungen, welche sich natürlich auch in einer Privilegiendiskrepanz der männlichen und weiblichen Angehörigen der Samuraifamilien zeigen. Demselben Stand zuzugehören bedingte also nicht automatisch den Zugang zu denselben Vorrechten. Zweitens schlug sich dieser Umstand auch in der, für diese Arbeit zur Verfügung stehenden, Literatur nieder, die bei der Schilderung der Samurai-Privilegien auf einen männlichen Bezugspunkt verweist.

3 Reinhard Zöllner, Geschichte Japans. Von 1800 bis zur Gegenwart, Paderborn 2006.

4 Gerhard Krebs, Das moderne Japan 1868-1952. Von der Meiji-Restauration bis zum Friedensvertrag von San Francisco (Oldenbourg Grundriss der Geschichte 36), München 2009.

5 Josef Kreiner (Hrsg.), Geschichte Japans, Stuttgart $2018^{6}$. 
zusammenfasst, ${ }^{6}$ eine grundlegende Stütze der Arbeit. Weitere seiner Publikationen zum Samuraistand, die ebenfalls herangezogen wurden, zeichnen ihn wohl als dessen besten Kenner im deutschsprachigen Raum aus. Ergänzend zu den Ausführungen Schwentkers wurden zur Erläuterung spezifischer Aspekte, wie etwa dem Bildungsstand der Samurai oder ihrer Rolle in der Administration, weitere Studien herangezogen. Besonders erwähnt sei hier der Aufsatz von Douglas R. Howland. ${ }^{8}$

\section{Soziologische Grundlagen auf Basis der Werke Pierre Bourdieus}

Um die Stellung der Samurai in der japanischen Gesellschaft beschreiben zu können, wird im Folgenden auf das Gedankengut des französischen Soziologen Pierre Bourdieu zurückgegriffen, das dieser in seinen Werken „Die verborgenen Mechanismen der Macht" ${ }^{\prime 9}$ und "Praktische Vernunft" ${ }^{\prime 10}$ darlegt.

Dabei stellt Bourdieu zunächst klar, dass die Strukturen der gesellschaftlichen Welt durch verschiedene Formen von Kapital bestimmt werden. Den Begriff Kapital selbst definiert er dabei als „akkumulierte Arbeit, entweder in Form von Material oder in verinnerlichter, ,inkorporierter' Form"11. Im Gegensatz zu dem in der Ökonomie verwendeten Kapitalbegriff beschränkt sich Bourdieus Definition nicht nur auf den profitorientierten Warenaustausch, sondern möchte alle Varianten des sozialen, nicht-ökonomischen Austausches miteinbeziehen. ${ }^{12}$ Insgesamt unterscheidet er zwischen drei grundlegenden Kapitalformen: dem ökonomischen, dem kulturellen und dem sozialen Kapital. Im Hinblick auf die soziale Funktion der unterschiedlichen Kapitalarten verwendet Bourdieu auch den Begriff des symbolischen Kapitals. ${ }^{13}$ Die Akkumulation respektive das Verhältnis zwischen diesen Kapitalformen, die auch ineinander umgewandelt werden können, bestimmen die Position von Individuen oder bestimmten Gruppen im sozialen Raum.

\subsection{Sozialer Raum und Feldtheorie}

Sowohl Individuen als auch Gruppen konstituieren sich durch ihre (kapitalbedingten) Unterschiede und nehmen nach Bourdieu dadurch "relative Positionen in einem [unsichtbaren] Raum von Relationen"14 ein. Er distanziert sich mit dieser Aussage von

$6 \quad$ Wolfgang Schwentker, Die Samurai im Zeitalter der Meiji-Restauration. Elitenwandel und Modernisierung in Japan, 1830-1890, in: Geschichte und Gesellschaft 28 (2002), Heft 1, S. 33-70.

$7 \quad$ Wolfgang Schwentker, Diener und Herrscher. Die japanische Geschichte im Spiegel der Samurai, in: Historisches Museum der Pfalz Speyer (Hrsg.), Samurai. Begleitbuch zur Ausstellung „Samurai“ im Historischen Museum der Pfalz Speyer, Ostfildern 2008, S. 15-29; Wolfgang Schwentker, Samurai und Soldaten. Zum Wandel des Militärs im Japan der Restaurationszeit, 1853-1895, in: Thomas Kolnberger (Hrsg.), Krieg und Akkulturation (ExpansionInteraktion-Akkulturation 5), Wien 2004, S. 130-152.

8 Douglas R. Howland, Samurai Status, Class, and Bureaucracy. A Historiographical Essay, in: The Journal of Asian Studies 60 (2001), Heft 2, S. 353-380.

$9 \quad$ Pierre Bourdieu, Die verborgenen Mechanismen der Macht (Schriften zu Politik \& Kultur 1), Hamburg 1992.

10 Pierre Bourdieu, Praktische Vernunft. Zur Theorie des Handelns, Frankfurt am Main 1998.

11 Bourdieu, Die verborgenen Mechanismen, S. 49.

12 Ebd., S. 49-50

13 Oliver Dimbath, Einführung in die Soziologie, o. O. 2016³, S. 298.

14 Bourdieu, Praktische Vernunft, S. 48. 
geschichtsphilosophischen Deutungen, wie dem marxistischen Kampf zwischen statischen Klassen und hebt stattdessen die Relativität von sozialen Positionen oder Praktiken hervor. ${ }^{15}$

Zur Veranschaulichung greift Bourdieu auf historische Beispiele, wie den Boxsport, zurück. Dieser war gegen Ende des 19. Jahrhunderts noch ein beliebter Aristokratensport in Frankreich, wurde allerdings von den Adeligen aufgegeben, als er von anderen Bevölkerungsschichten - hauptsächlich dem Bürgertum - nach und nach übernommen wurde. ${ }^{16}$ Während die bürgerlichen Schichten also versuchten sich dem Adel durch die Übernahme seiner Praktiken anzunähern, in der Hoffnung damit den sozialen Unterschied zwischen den Gruppen zu verringern, distanzierten sich die Aristokrat*innen zugleich davon, um die bestehende Standesdifferenz aufrecht erhalten zu können. Bourdieu stellt fest, dass sich die Distinktionsmerkmale sozialer Gruppen je nach Zeitraum sowie dem Angebot an Gütern und Praktiken definieren. "Vorgefertigte" Klassen mit bestimmten Merkmalen sind laut Bourdieu daher keine festen Größen in der Geschichte. Wie aber schon das Boxsport-Beispiel zeigt, bleibt der Kern der Klassen-Idee die angestrebte soziale Differenzierung von Individuen oder Gruppen zu erhalten. Auf diesen Ausführungen aufbauend schlägt Bourdieu vor, weniger von Klassen an sich, sondern vielmehr von sozialen Räumen zu sprechen, in denen sich Klassen abgrenzen lassen. ${ }^{17}$

Wenn Gesellschaften nun als soziale Räume - also als Strukturen von Unterschieden - wahrgenommen werden, ist es notwendig, die objektiven Prinzipien, welche hinter der Konstruktion von sozialen Unterschieden stehen, zu identifizieren. Für Bourdieu ist jenes zentrale Prinzip die Distributionsstruktur der bereits erwähnten Kapitalsorten. ${ }^{18}$ Der soziale Raum wird in der Terminologie Bourdieus damit zu einem großen „Feld” (im Sinne eines Kraft- oder auch Kampffeldes) ${ }^{19}$, das wiederum mehrere "soziale Felder"20 beinhaltet. Um das jeweilige soziale Feld zu beherrschen, versuchen die agierenden Akteur*innen sich innerhalb des Feldes zu profilieren und ihre Verschiedenheit zu anderen Akteur*innen hervorzuheben. Hierbei kommen zwei Möglichkeiten in Frage: Entweder durch die Anhäufung von Kapital oder indem die Regeln des Kapitalbedarfs geändert werden. Insgesamt bieten diese Überlegungen Bourdieus die Grundlage, ökonomische wie kulturelle Konflikte in den unterschiedlichen Feldern des sozialen Raumes beschreiben zu können. ${ }^{21}$ Für diesen Schritt müssen allerdings erst die bereits angesprochenen Kapitalsorten genauer erläutert werden.

Bourdieu, Praktische Vernunft, S. 48.

Ebd., S. 17.

Ebd., S. 48-49.

Bourdieu, Praktische Vernunft, S. 49

Ebd.

Bourdieu untersuchte unter anderem das künstlerische, literarische, wissenschaftliche und religiöse Feld: Pierre Bourdieu/Loïc Wacquant, Reflexive Anthropologie, Frankfurt am Main 1996, S. 124.

21 Dimbath, Einführung, S. 294-297. 


\subsection{Kapitalformen bei Bourdieu}

Bourdieu nennt das ökonomische, kulturelle, sowie soziale Kapital als die drei grundlegenden Kapitalarten. Ersteres ist mitunter selbsterklärend: Unter dem ökonomischen Kapital fasst Bourdieu all das zusammen, was sich unmittelbar in Geld konvertieren lässt, womit meist Eigentum gemeint ist.22 Zur Beschreibung der Funktionsweisen von sozialen Räumen, die sich nicht nur aus der ökonomischen Potenz ihrer Akteure heraus erklären lassen, führt er weitere Kapitalbegriffe ein.

\subsubsection{Kulturelles Kapital}

Auch hier unterscheidet Bourdieu wiederum zwischen drei Formen: Zum Ersten kann das kulturelle Kapital in einem körpergebundenen, inkorporierten Zustand existieren. Zentral ist hierbei der Erwerb von Bildung, die für Bourdieu die „Akkumulation von Kultur in korporiertem Zustand “23 darstellt. Ihrer Aneignung geht ein Verinnerlichungsprozess in Form von Unterrichts- oder Lernzeit voraus, die persönlich investiert werden muss und sich nicht durch fremde Personen vollziehen lassen kann. Damit in Verbindung steht auch die familiäre (Primär-)Erziehung, die diesen Bildungsprozessen vorausgeht und sich positiv oder negativ auf den Erwerb von kulturellem Kapital, beispielsweise in der Schule, auswirken kann. Im Unterschied zu den anderen Kapitalarten, die sich in Geld (ökonomisches Kapital) oder Adelstiteln (soziales Kapital) manifestieren, kann das inkorporierte kulturelle Kapital weder durch Schenkung, Vererbung, Kauf oder Tausch kurzfristig weitergegeben werden. ${ }^{24}$ Da die Aneignung von kulturellem Kapital aber eine gewisse Zeit erfordert, darf das vorhandene ökonomische Kapital bei dessen Erwerb nicht ganz außer Acht gelassen werden: Ein Individuum kann die Zeitspanne für die Akkumulation von kulturellem Kapital nämlich nur so lange ausdehnen, wie ihm seine Familie eine von ökonomischen Zwängen befreite Zeit garantieren kann. ${ }^{25}$

Als zweite Form nennt Bourdieu das „objektivierte Kulturkapital”, welches im Gegensatz zum inkorporierten materiell übertragbar ist. Schriften, Gemälde, Denkmäler, Instrumente oder auch Maschinen können dabei als Träger fungieren. Einzelne Personen müssen allerdings über bestimmte kulturelle Fähigkeiten - also inkorporiertes kulturelles Kapital - verfügen, um das objektivierte Kulturkapital für sich nutzbar zu machen. Dies stellt die Voraussetzung dar, um den Genuss eines Gemäldes oder den Gebrauch einer Maschine überhaupt erst möglich zu machen. Insgesamt kann objektiviertes Kulturkapital zwar mit ökonomischem Kapital erworben werden, verlangt für seine Nutzbarmachung aber nach Personen mit inkorporiertem Kulturkapital. ${ }^{26}$

Zuletzt zählt Bourdieu noch das „institutionalisierte Kulturkapital“ in Form von schulischen oder akademischen Titeln auf. Diese dienen ihren Träger*innen als Nachweis ihres kulturellen Kapitals - unabhängig davon, ob sie dieses tatsächlich zu einem ge-

Bourdieu, Die verborgenen Mechanismen, S. 52.

Ebd., S. 55.

Bourdieu, Die verborgenen Mechanismen, S. 55-56.

Ebd., S. 58-59.

Ebd., S. 59. 
gebenen Zeitpunkt besitzen. Diese institutionelle Anerkennung von Kulturkapital hebt sie von Autodidakten, welche sich stets beweisen müssen, ab. ${ }^{27}$

\subsubsection{Soziales Kapital}

Als „Ressourcen, die auf der Zugehörigkeit zu einer Gruppe beruhen“28, fasst Pierre Bourdieu das „soziale Kapital“ zusammen. Das Gesamtkapital der einzelnen Gruppenmitglieder dient dabei allen gemeinsam als Sicherheit. Praktische Grundlagen von Sozialkapital-Beziehungen sind einerseits materielle oder symbolische Tauschbeziehungen sowie andererseits gesellschaftliche Institutionalisierungsakte, die beispielsweise mit der Übernahme eines Namens, der die Zugehörigkeit zu einer bestimmten Familie oder Klasse ausdrückt, erfolgen können. Der Umfang des sozialen Kapitals von einzelnen Akteur*innen hängt wiederum von der tatsächlichen Ausdehnung ihres Beziehungsnetzwerks und dem verfügbaren (ökonomischen oder kulturellen) Kapital seiner Partner*innen innerhalb dieses Netzwerks ab. ${ }^{29}$

Dass solche Beziehungsnetzwerke überhaupt existieren, führt Bourdieu nicht auf irgendwelche Gegebenheiten, die durch einen ursprünglichen Institutionalisierungsakt geschaffen wurden, zurück, ${ }^{30}$ sondern geht vielmehr von einer fortlaufenden Institutionalisierungsarbeit aus. Diese ist notwendig, um dauerhafte, nützliche Beziehungen zu produzieren oder aufrecht zu erhalten. Beziehungsnetze sind also Produkte individueller oder kollektiver Investitionsstrategien, deren Ziel die Schaffung und Erhaltung von nützlichen Sozialbeziehungen ist. Verpflichtungen, die sich aus diesen Beziehungen ergeben, können auf subjektiven Gefühlen wie Anerkennung, Freundschaft oder Respekt beruhen, oder auch auf institutionellen Garantien (Rechtsansprüchen) basieren. ${ }^{31}$

Das gesamte soziale Kapital einer Gruppe wird nach Bourdieu durch „Delegationen“, welche im Namen der jeweiligen Gruppe handeln, vertreten. Als Beispiel für familiäre Gruppen wäre hier das Familienoberhaupt zu nennen. Ihnen fällt des Weiteren die Aufgabe zu, die Gruppenehre zu bewahren, indem äußere Feinde abgewehrt und Gruppenmitglieder, welche Verfehlungen begangen haben, zurechtgewiesen werden. ${ }^{32}$

\subsection{Das Feld der Macht}

Als Letztes sei noch der Terminus des „Feldes der Macht" erwähnt, welcher in gewisser Weise alle bisherigen Ausführungen nochmals zusammenfasst und als Ausgangspunkt des folgenden Kapitels dienen soll. Das Feld der Macht, welches nicht mit dem politischen Feld zu verwechseln ist, hebt sich von den bisher beschriebenen Feldern wesentlich ab:

27 Bourdieu, Die verborgenen Mechanismen, S. 61-62.

28 Ebd., S. 63

29 Ebd., S. 64

30 Wie etwa die genealogische Definition von Verwandtschaftsbeziehungen im Falle der Familie: Ebd., S. 65.

31 Ebd.

32 Ebd., S. 67-68. 
„Es ist der Raum der Machtverhältnisse zwischen verschiedenen Kapitalsorten oder, genauer gesagt, zwischen Akteuren, die in ausreichendem Maße mit einer der verschiedenen Kapitalsorten versehen sind, um gegebenenfalls das entsprechende Feld beherrschen zu können, und deren Kämpfe immer dann an Intensität zunehmen, wenn der relative Wert der verschiedenen Kapitalsorten [...] ins Wanken gerät; [...]."33

Entscheidender Faktor ist laut Bourdieu also der Erhalt beziehungsweise die Veränderung des „Wechselkurses“ zwischen den verschiedenen Kapitalsorten, der mit der Macht über bürokratische Instanzen einhergeht. Beispielsweise können so administrative Maßnahmen gesetzt werden, um auf die Seltenheit von Bildungstiteln, welche den Zugang zu herrschenden Positionen eröffnen, einzuwirken. ${ }^{34}$ Das mit ihnen verbundene institutionalisierte kulturelle Kapital würde an Wert gewinnen oder verlieren und bestimmte Personengruppen dadurch profilieren. Inwiefern dies auf das soziale Kapital des Samuraistandes zutrifft, dessen Wechselkurs sich mit der Einführung des modernen japanischen Verfassungsstaates wesentlich veränderte, soll im Folgenden aufgezeigt werden.

\section{Japan am Ende der Frühen Neuzeit}

\subsection{Periodisierung}

Den sozialen Raum Japans in der Frühen Neuzeit, in welchem sich das Feld der Macht konstituierte, teilt Reinhard Zöllner am Beginn seiner Monografie zwischen drei konkurrierenden Akteuren auf: der Familie der Tokugawa, dem Hof des japanischen Kaisers sowie der Gruppe der japanischen Fürstentümer. ${ }^{35}$ Alle drei spielen im Hinblick auf die Epochengliederung Japans eine maßgebliche Rolle, die nun näher erläutert werden soll.

Hierzu muss zunächst das gewohnte Terrain der eurozentristischen Geschichtsperiodisierung, welche die Epochengrenze zwischen Mittelalter und Neuzeit aufgrund eines Bündels von mittel- und langfristig einschneidenden Veränderungen meist um die Wende vom 15. ins 16. Jahrhundert zieht, ${ }^{36}$ verlassen werden. Dasselbe gilt für die Binnendifferenzierung zwischen Früher und Später Neuzeit - ein Bruch, der auch im Mittelpunkt dieser Arbeit steht.

Die Frühe Neuzeit wird in Japan im Allgemeinen mit jenem Zeitraum zwischen 1603 und 1867 gleichgesetzt, in welcher die Stadt Edo ${ }^{37}$ als Residenz der regierenden Familie der Tokugawa diente. ${ }^{38} \mathrm{Ihr}$ ging zwischen dem 12. und 16. Jahrhundert eine Periode

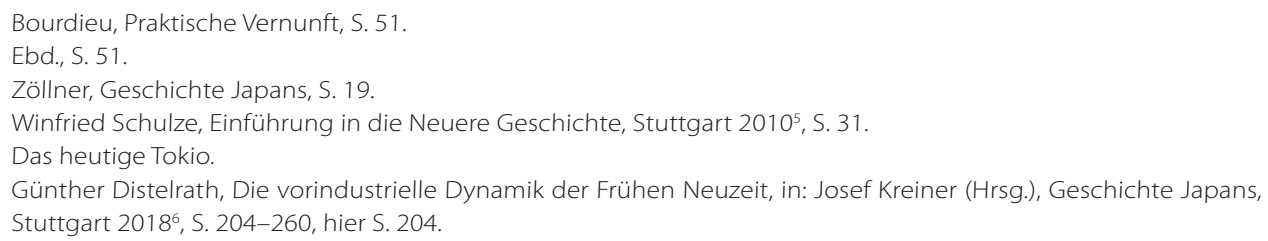


voraus, in welcher der Kaiser (tennō) seine politische Machtbasis größtenteils einbüßte und an die, von verschiedenen Herrscherfamilien eingerichteten, Shogunate verlor. Bei diesen handelte es sich grob gesprochen um von einem Militärregenten (shōgun) geführte Regierungen, deren ursprüngliche Aufgabe in der Herrschaftssicherung des Kaisers bestand. Historisch existierten drei Shogunate unter den Familien der Minamoto (1192-1333), Ashikaga (1336-1573) sowie den bereits erwähnten Tokugawa. Während die Minamoto die ihnen übertragene Militär- und Polizeigewalt zum Aufbau eigener Herrschaftsstrukturen auf Kosten des Kaiserhofes einleiteten und damit das erste der Shogunate schufen, erodierte diese Ordnung unter der Herrschaft der Ashikaga-Familie, die ihre Macht weitgehend an die einzelnen Lehensfürsten (daimyō) verlor. ${ }^{39}$ Unter dem letzten der Shogunate, das von der Familie der Tokugawa begründet wurde, kam es ab dem 17. Jahrhundert zu einer Re-Zentralisierung im Rahmen eines Feudalstaates, der bis in die zweite Hälfte des 19. Jahrhunderts bestand. ${ }^{40}$

Das Jahr 1868 gilt unter den Fachautor*innen gemeinhin als Zäsur, ${ }^{41}$ die die Späte Neuzeit einläutet, was hauptsächlich mit dem Prozess der Wiederherstellung des politischen Machtmonopols des Kaisers, der sogenannten Meiji-Restauration, ${ }^{42}$ zusammenhängt. In Verbindung mit der Restaurationsperiode sind aus historiografischer Sicht vier wesentliche Einschnitte zu nennen: das Ende der über 250 Jahre währenden Hegemonie der bis dahin herrschenden Tokugawa-Familie und den daraus resultierenden Folgen wie die Abschaffung des Feudalsystems, welche mit der Gründung des japanischen Nationalstaats einhergeht, dem damit entstehenden nationalen Einheitsgefühl der japanischen Bevölkerung sowie die Umbenennung des Herrschaftssitzes ${ }^{43}$ von Edo in Tokio. ${ }^{44}$ Die Hintergründe der Meiji-Restauration sowie deren Einfluss auf die soziale Ordnung Japans, insbesondere der Stellung der Samurai, werden im nachfolgenden Abschnitt näher behandelt. Zunächst sollen jedoch die Entwicklungslinien bis zur Mitte des 19. Jahrhunderts detaillierter geschildert werden.

\subsection{Gesellschaftsordnung}

\subsubsection{Die Herrschaft der Tokugawa}

Mit ihrem Triumph in der Schlacht von Sekigahara im Jahr 1600 waren die Tokugawa siegreich aus einer militärischen Konfrontation zwischen den westlichen und östlichen

39 Regine Mathias, Shogunat, in: Enzyklopädie der Neuzeit, http://dx.doi.org/10.1163/2352-0248_edn_COM_348940, eingesehen 2.2.2020.

40 Kazuhiro Takii/Michael Wachutka (Hrsg.), Staatsverständnis in Japan. Ideen und Wirklichkeiten des japanischen Staates in der Moderne (Staatsverständnisse 90), Baden-Baden 2016, S. 12.

41 Krebs, Das moderne Japan, S. 1-7; Zöllner, Geschichte Japans, S. 140-177; Sven Saaler, Die Bedeutung der Epochenmarke 1868 in der Japanischen Geschichte. Restauration, Revolution, Reform, in: Saeculum 56 (2005), Heft 1, S. 69-104.

42 Siehe Kapitel 4

43 Dies ist vor allem historiografisch nicht unwesentlich, da die Shogunatsperioden in der japanischen Geschichtsschreibung jeweils nach ihrem Amtssitz (z. B. Edo-Zeit respektive Edo-bakufu) benannt werden: Mathias, Shogunat.

44 Einen sinnvollen Überblick gibt hier die Tabelle zur Epochengliederung in dem von Josef Krainer herausgegebenen Sammelband zur Geschichte Japans: Kreiner (Hrsg.), Geschichte Japans, S. 478-479. 
Kriegsherren Japans hervorgegangen und konnten so den Grundstein ihrer Macht legen. Als Oberbefehlshaber der östlichen Armeen konnte ihr Familienoberhaupt, Tokugawa leyasu ${ }^{45}$, in den folgenden Jahren eine stabile Herrschaft etablieren. ${ }^{46} 1603$ ließ er sich vom Kaiser den Titel des shōgun, welcher ursprünglich aus China stammte und in etwa mit "Generalissimus zur Vertreibung der Barbaren"47 übersetzt werden kann, verleihen. Auf die Theorien Pierre Bourdieus übertragen, lässt sich der shōgunTitel in die Kategorie des institutionalisierten Kulturkapitals einordnen und unterscheidet sich damit vom Adelstitel, den Bourdieu zum sozialen Kapital zählt, da dessen Erringung einen Standesübergang markiert, der seinen Träger*innen den Zugang zu neuen Beziehungsnetzwerken ermöglicht, an welchen Tokugawa leyasu als Adeliger aber bereits teilhatte. Die Verleihung des shōgun-Titels zeichnet hingegen eine Kulturleistung, nämlich die Aneignung und Anwendung militärischer Kenntnisse, aus.

Seit dem 12. Jahrhundert waren mit diesem Titel eine Reihe von juridischen, politischen und diplomatischen Vorrechten gegenüber den übrigen Fürstenhäusern, aber auch gegenüber dem Kaiserhof verbunden. Der Herrschaftsanspruch, welcher damit verbunden war, zeigt sich in der zeitgenössischen Anrede für den shögun. Diese lautete kōgi, was so viel wie „öffentliche Gewalt"48 bedeutete..9 Mit diesen Privilegien ausgestattet, trieben die Tokugawa-Herrscher einen Umbau der Gesellschaft nach ihren Vorstellungen voran. Zur Erweiterung ihres ökonomischen Kapitals wurden die großen Edelmetallminen des Landes sowie die Städte Osaka und Nagasaki unter die direkte Verwaltung der sich entwickelnden Zentralregierung gestellt.50 Weitere stabilisierende Maßnahmen in diese Richtung stellten die Vereinheitlichung von Gewichten und Maßen sowie des Geldsystems dar. ${ }^{51}$ Tokugawa leyasu nutzte auch sein bereits bestehendes soziales Kapital, um seine Position zu sichern. Während er diejenigen Fürsten, welche ihm feindlich gesinnt waren, in weit entfernte Regionen versetzte, belehnte er seine bisherigen Verbündeten sowie Fürsten, mit denen er verwandt war, mit den Kerngebieten um Edo. ${ }^{52}$

In den folgenden Jahrzehnten etablierte sich der sogenannte baku-han-Staat. Dabei kam es zur dualistischen Herrschaftsteilung zwischen der zentralen Shogunatsregierung, dem bakufu ${ }^{53}$, und den über 250 Fürstentümern (han), die allerdings unter der Oberhoheit des shōgun standen. Gemeinsam mit der hierarchischen Gliederung der Bevölkerung in vier Stände - Samurai, Bauern, Handwerker und Kaufleute - wurde die Herrschaft der Fürsten mit dem shōgun an der Spitze als Entsprechung kosmischer

45 Die in der Arbeit aufscheinenden Personennamen werden in der in Japan üblichen Form angegeben, wobei der Familien- oder Geschlechtername dem persönlichen Vornamen vorangestellt wird: Kreiner (Hrsg.), Geschichte Japans, S. 24.

46 Distelrath, Die vorindustrielle Dynamik, S. 213.

47 Mathias, Shogunat.

48 Zöllner, Geschichte Japans, S. 21.

49 Ebd., S. 20-21.

50 Distelrath, Die vorindustrielle Dynamik, S. 215.

51 Mathias, Shogunat.

52 Distelrath, Die vorindustrielle Dynamik, S. 213-215.

53 Dieser Begriff, der eigentlich das Feldherrnlager eines shōgun bezeichnete, bürgerte sich im 19. Jahrhundert ein und stellte eine abwertende sowie delegitimierende Bezeichnung für die Tokugawa-Herrschaft dar. Damit stand sie auch im Widersinn zur Selbstbezeichnung des shögun als kōgi: Zöllner, Geschichte Japans, S. 24. 
Gesetze, die sich aus dem Konfuzianismus ableiteten, gesehen und damit (im weiteren Sinne) naturrechtlich begründet. Jedem Stand wurden dabei exklusive ökonomische Tätigkeiten zugewiesen, wobei die Verwaltungsaufgaben den Samurai zufielen. Die Übertretung der Standesgrenzen bedeutete nach dieser Weltanschauung zugleich eine Verletzung der kosmischen Harmonie. ${ }^{54}$ Das Kaiserhaus, dessen Hofadel (kuge) und ohnehin diskriminierte Randgruppen ${ }^{55}$ wurden von dieser Einteilung nicht erfasst. ${ }^{56}$

\subsubsection{Die Fürstentümer}

Die föderale Natur des baku-han-Staats ergab sich aus der nicht unwesentlichen Rolle der einzelnen Feudalherrscher Japans, auf die das bakufu zur indirekten Machtausübung angewiesen war. Der Status eines Fürsten (daimyō), der ebenfalls der sozialen Schicht der Samurai angehörte, ${ }^{57}$ stand dabei in Verbindung mit seinem ökonomischen Kapital. Nur diejenigen Vasallen der Tokugawa, deren Erträge mindestens 10.000 koku ${ }^{58}$ Reis pro Jahr betrugen, zählten zu den daimyō und konnten die damit verbundenen Rechte - wie etwa das Audienzrecht beim shōgun - in Anspruch nehmen. Weitere Distinktionsmerkmale, welche die Hierarchie der Fürsten festlegten, waren häufig symbolischer Art. Dabei zählte zum Beispiel, ob eine der alten kaiserlichen Provinzen Teil ihres Herrschaftsgebietes war oder welches Audienzzimmer ihnen in der Burg Edo zugewiesen ${ }^{59}$ wurde. $^{60}$

Eine im Jahr 1635 eingeführte Pflicht brachte die daimyō und ihr Gefolge allerdings dazu, auch außerhalb von Audienzgesuchen alle zwei Jahre in der Hauptstadt Edo zu residieren, während ihre Familien ständig dort leben mussten. Damit wurde eine Praxis des 15. Jahrhunderts weitergeführt, bei der Vasallen zur Gewährleistung ihrer Loyalität Geiseln an ihre jeweiligen Feudalherren übergaben. Das ständige Kommen und Gehen bedeutete zugleich, dass ein daimyō stets über die Erlässe des shōgun informiert war und diese daher auch bekanntmachen und umsetzen musste. ${ }^{61}$ Die wechselnde Residenzpflicht war vor allem für die, dem shōgun nahestehenden Fürstentümer mit einem erheblichen finanziellen Aufwand verbunden und bildete in Summe zwischen siebzig und achtzig Prozent ihrer Ausgaben. ${ }^{62}$ Dieses System der alternierenden Residenzen

54 Distelrath, Die vorindustrielle Dynamik, S. 220.

55 Diese ausgestoßenen Gruppen, welche als eta (viel Schmutz), burakumin (Dorfbewohner) oder hinin (NichtMenschen) bezeichnet wurden, unterschieden sich nicht ethnisch oder kulturell von der Restbevölkerung, übten aber Tätigkeiten aus, die mit Schmutz, Blut oder Leichen zu tun hatten, wie beispielsweise Bestatter, Henker oder auch Gerber: Krebs, Das moderne Japan, S. 3.

56 Distelrath, Die vorindustrielle Dynamik, S. 214-215.

57 John Whitney Hall, Das japanische Kaiserreich (Fischer Weltgeschichte 20), Frankfurt am Main 200915, S. 178.

58 Da der Großteil der Tribute und Steuern zu Beginn des Tokugawa-Shogunats noch in Naturalien geleistet wurde und Reis das wertvollste Anbauprodukt war, wurde das Standard-Hohlmaß für ungeschälten Reis, das koku, als Rechnungseinheit eingesetzt. 1 koku entspricht 180 Litern, also ungefähr 150 kg Reis: Zöllner, Geschichte Japans, S. 34-35.

59 Die Bedeutung des Raumes für die Konstitution und Wahrnehmung sozialer Rangordnungen war in den letzten Jahren Teil intensiver Forschungsdebatten: Christoph Dartmann/Marian Füssel/Stephanie Rüther (Hrsg.), Raum und Konflikt. Zur symbolischen Konstituierung gesellschaftlicher Ordnung in Mittelalter und Früher Neuzeit, Münster 2004.

60 Zöllner, Geschichte Japans, S. 36-37.

61 Hall, Das japanische Kaiserreich, S. 169.

62 Zöllner, Geschichte Japans, S. 45. 
sicherte den Einfluss des shōgun zusätzlich ab, spiegelte aber gleichzeitig die Furcht vor einem Rückfall in jene kriegerischen Zustände, wie sie vor dem Herrschaftsantritt der Tokugawa bestanden hatten, wider. ${ }^{63}$

\subsubsection{Der Hof des Kaisers}

Die Begründung des Kaiserhauses respektive das Amt des tennō ${ }^{64}$, das im Deutschen als „Kaiser" übersetzt wird, soll legendären Erzählungen nach auf das Jahr 660 v. Chr. zurückgehen. Seine Legitimität als Herrscher leitete der tennō aus einer postulierten göttlichen Abstammung ab, auf welcher auch die politische Macht und die geistliche Autorität des Kaiserhauses fußte. In Verbindung mit der einzigartigen priesterlichen Funktion des Kaisers spricht Michael Wachutka auch von einem sakralen Königtum. ${ }^{65}$ Faktisch gelang es dem tennō im 8. Jahrhundert erstmals eine Zentralregierung mit Sitz in Kyoto zu etablieren und den Frieden im Land bis ins 12. Jahrhundert zu sichern. Die militärische Macht der kaiserlichen Zentralregierung, die innerhalb dieser Friedensperiode auf ein Minimum schrumpfte, war daraufhin nicht mehr in der Lage den kriegerischen Auseinandersetzungen des (Schwert-)Adels Einhalt zu gebieten, wodurch sich die Vorherrschaft des tennō nur noch auf den geistig-kulturellen Bereich beschränkte. ${ }^{66}$ Ungeachtet seiner politischen Machtlosigkeit hatte dieser seine nominelle Autorität als göttlicher Herrscher niemals komplett eingebüßt, weshalb sich die Shogune trotz ihrer Allmacht stets durch den Kaiser bestätigen ließen. ${ }^{67}$

Mit dem Beginn der Tokugawa-Regierungsperiode wurde vom Kaiserhof erwartet, die Politik des Systems symbolisch abzusegnen. Im Austausch für die Übernahme aller Schulden, welche durch die kaiserliche Hofaristokratie verursacht wurden, setzte sich der tennō dem shōgun nicht zur Wehr. Kurz nach dem Beginn seines Shogunats verabschiedete Tokugawa leyasu 1611 ein Gesetz für den Hofadel, das diesem die intensive Beschäftigung mit den damaligen „Wissenschaften“ - gemeint waren Künste wie Poesie, Kalligrafie oder Malerei - sowie das strikte Einhalten eines Hofzeremoniells unter Androhung von schweren Strafen vorschrieb. Dies hatte eine Isolation des Kaisers und seiner Hofadeligen in ihren Palästen zur Folge. Zudem unterband der shōgun jeglichen Kontakt zwischen dem Kaiser und anderen Fürsten oder Vasallen, indem Audienzen ausschließlich über die Regierung in Edo abgewickelt wurden. ${ }^{68}$ Sein

63 Distelrath, Die vorindustrielle Dynamik, S. 218

64 Übersetzt: „himmlischer Herrscher“: Michael Wachutka, Der kokutai-Diskurs im Staatsverständnis des 19. und frühen 20. Jahrhunderts. Kerngedanken und Entwicklung der Idee von Japans einzigartigem Nationalwesen, in: Kazuhiro Takii/Michael Wachutka (Hrsg.), Staatsverständnis in Japan. Ideen und Wirklichkeiten des japanischen Staates in der Moderne (Staatsverständnisse 90), Baden-Baden 2016, S. 65-88, hier S. 68.

65 Ebd., S. 68-69.

66 Nagao Ryūichi, Der Staat Japan in historischer Perspektive, in: Kazuhiro Takii/Michael Wachutka (Hrsg.), Staatsverständnis in Japan. Ideen und Wirklichkeiten des japanischen Staates in der Moderne (Staatsverständnisse 90), Baden-Baden 2016, S. 13-45, hier S. 21-22.

67 Wachutka, Der kokutai-Diskurs, S. 69

68 Methoden, um Kontakte zu Herrscher*innen zu regulieren, finden sich zur selben Zeit auch in England: Ronald G. Asch, "The Politics of Access". Hofstruktur und Herrschaft in England unter den frühen Stuarts, 1603-1642, in: Werner Paravicini (Hrsg.), Alltag bei Hofe. 3. Symposium der Residenzen-Kommission der Akademie der Wissenschaften in Göttingen, Ansbach, 28. Februar bis 1. März 1992 (Residenzenforschung 5), Sigmaringen 1995, S. 243-266. 
soziales Kapital konnte der Kaiser daher nur durch den Kontakt zu Künstlern oder anderen Berufsgruppen, die sich außerhalb des Feldes der Macht befanden, erweitern. Eine Ausnahme stellten hier wenige Angehörige großer Fürstenhäuser dar, die die Töchter der Kaiser als Bräute für ihre Nachkommen ins Auge gefasst hatten.69

Unter den Umständen einer solchen Machtverteilung mag es nicht verwunderlich erscheinen, dass europäische Japan-Reisende der Edo-Zeit, vor allem Geistliche und Ordensmänner, die ihnen bekannten sozialen Kategorien auf die japanische Gesellschaft anwandten und dadurch den shögun als Kaiser betrachteten, den tennō hingegen als eine Art Papst ansahen. ${ }^{70}$

\subsection{Die Stellung der Samurai in der sozialen Ordnung Japans}

Neben den Vergleichen von Papst und Kaiser mit tennō und shōgun, wurden in der historischen Forschung ${ }^{71}$ sowie auf der populär(wissenschaftlich)en Ebene ${ }^{72}$ immer wie- $^{-}$ der Parallelen zwischen europäischen Rittern und japanischen Samurai gezogen. Der Begriff bushi - ein im Japanischen geläufigerer Ausdruck für die Samurai - umfasst das Tragen sowie den ehrenhaften Gebrauchen von Waffen. Dem Samurai, der als "Diener" übersetzt werden kann, kommt daher eine Rolle als Bewahrer der Ordnung zu, die aber über eine rein militärische Funktion hinausgeht. Der, diesem Ideal entsprechende bushi wurde also nicht nur als Krieger, sondern auch als moralischer Erzieher betrachtet. ${ }^{73}$

Innerhalb des japanischen Mittelalters stellte der bushi zwar einen Aristokraten dar, der sich in seinen Aufgabenbereichen allerdings weit vom restlichen Hofadel abhob. Während Letztere sich in der kaiserlichen Hauptstadt abschotteten, lebten die Samurai in unmittelbarer Nähe zu den von ihnen verwalteten Ländereien und konzentrierten sich auf die Schulung ihrer kriegerischen Fähigkeiten. Das kulturelle Kapital dieses Standes umfasste also administrative sowie militärische Fähigkeiten, zu denen Bogenschießen, Fechten, Reiten und Menschenführung zählten. Die zwei Säulen, auf welchen das Selbstverständnis des bushi fußte, stellten einerseits die Loyalität gegenüber seinem Herrn - dem daimyō oder dem shōgun - sowie andererseits die Steigerung der eigenen Familienehre dar. ${ }^{74}$ Das verbreitete, stereotype Bild der Samurai als Kriegerfürsten wurzelt aber nicht in den eben beschriebenen Funktionen, sondern in den Machtkämpfen der Sengoku-Zeit (1467-1568), in welcher das Land in eine Vielzahl von regional beherrschten Einheiten zerfiel und sich für knapp hundert Jahre im Kriegszustand befand. ${ }^{75}$

69 Zöllner, Geschichte Japans, S. 46.

70 Sichtbar wird dies beispielsweise in jesuitischen Reiseberichten: David Spafford, Emperor and Shogun, Pope and King. The Development of Japan's Warrior Aristocracy, in: Bulletin of the Detroit Institute of Arts 88 (2014), Heft 1, S. 10-19, hier S. 11.

71 Reinhard Zöllner, Samurai und Ritter, in: Historisches Museum der Pfalz Speyer (Hrsg.), Samurai. Begleitbuch zur Ausstellung „Samurai“ im Historischen Museum der Pfalz Speyer, Ostfildern 2008, S. 173-179.

72 Jens Lubbadeh, Samurai-Schau. Das Geheimnis der todbringenden Ritter, in: Der Spiegel, 24.2.2008, https:// www.spiegel.de/wissenschaft/mensch/samurai-schau-das-geheimnis-der-todbringenden-ritter-a-536951.html, eingesehen 7.3.2020.

73 Schwentker, Diener und Herrscher, S. 15.

74 Hall, Das japanische Kaiserreich, S. 96-97.

75 Schwentker, Diener und Herrscher, S. 23. 
Nach der neuerlichen Reichseinigung unter Tokugawa leyasu zementierte sich die gesellschaftliche Schlüsselstellung der Samurai. Vor allem die bereits erwähnten Gesetzeserlässe des Shogunats, die eine Einteilung der Gesellschaft in vier Stände zur Folge hatte, wobei der Kriegeraristokratie eine führende Position zugewiesen wurde, veränderte die soziale Mobilität in Japan grundsätzlich. Anders als in der Phase der Konsolidierungskriege war es nun nicht mehr möglich, Eingang in den Samurai-Stand zu finden, welcher die Herrschaft der Zentralregierung in Edo nun als multifunktionale Elite stützte. ${ }^{76}$ Jedoch wäre es auch falsch, die Samurai als homogene Gruppe zu betrachten. Durch ihren - im Vergleich zum europäischen Adel - relativ hohen Anteil von etwa fünf bis sechs Prozent an der Gesamtbevölkerung ergab sich eine starke Binnendifferenzierung je nach Ämtern, Besitzrechten und Rang. Die Spanne reicht dabei von den daimyō selbst über deren berittenes Begleitpersonal bis hin zu einfachen Torwächtern. ${ }^{77}$ Nach außen zeigte sich der Status der Samurai aber grundsätzlich anhand des Privilegs einen Nachnamen zu führen und zwei Schwerter tragen zu dürfen. ${ }^{78}$

Beide Vorrechte verweisen auf die Standeszugehörigkeit der Samurai und sind daher zunächst dem sozialen Kapital zuzuordnen. Der Umstand, dass aber auch Ärzten sowie Priestern die Führung eines Familiennamens gestattet wurde, ${ }^{79}$ deutet diesbezüglich auf eine Verschränkung von sozialem und kulturellem Kapital hin. Betreffend des Tragens von Schwertern konnte aufgezeigt werden, dass dieses Privileg auch Stadtbewohnern sowie Bauern als Gegenleistung für außergewöhnliche Dienste gewährt wurde und diese damit vorübergehend ihren ursprünglichen sozialen Status überwinden konnten. ${ }^{80}$ Für jene Personen bedeutete dies also einen individuell-temporären Erwerb von sozialem Kapital, der ihre Herrschaftsnähe ausdrückte. Das bereits skizzierte Selbstverständnis des bushi, welches Loyalität gegenüber seinem Herrn sowie den ehrenhaften Gebrauch von Waffen einforderte, ermöglichte ihm hingegen permanenten Zugang zu dem, in diesem Standesrecht manifestierten, sozialen Kapital.

Allerdings forderte der Zugang zu Standesrechten auch Standespflichten ein. Trotz ihres formalen gesellschaftlichen Aufstiegs beschnitten die ständischen Verordnungen der Tokugawa-Regierung den bisherigen Freiraum der Samurai erheblich. Mit der strikten Trennung von Stand, Beruf und Lebensordnung wurde ihnen verboten den Beruf eines Bauern, Handwerkers oder Kaufmanns zu ergreifen. Bedingt durch die Trennung der Samurai von anderen sozialen Gruppen, vor allem den Bauern, wurden sie gezwungen ihre ländlichen Domizile aufzugeben und in die Burgstädte ihrer Herren zu ziehen. ${ }^{81}$ Daraus resultierte ein unglaubliches Wachstum der Städte, in denen die Samurai zum Teil über die Hälfte der Einwohnerschaft ausmachten. Edo selbst wuchs bis zum 18. Jahrhundert zu einer Millionenstadt heran. ${ }^{82}$ Betreffend ihres ökonomischen

\footnotetext{
76 Hall, Das japanische Kaiserreich, S. 178.

77 Schwentker, Diener und Herrscher, S. 26.

78 Hall, Das japanische Kaiserreich, S. 178.

79 Zöllner, Geschichte Japans, S. 56.

80 Howland, Samurai Status, S. 361.

81 Schwentker, Die Samurai, S. 42.

82 Brett L. Walker setzt die Millionenmarke bereits im frühen 17. Jahrhundert an, Reinhard Zöllner hingegen für den Beginn des 18. Jahrhunderts: Brett L. Walker, A Concise History of Japan, Cambridge 2015, S. 125-126; Zöllner,
} 
Kapitals wurden die Krieger von der Shogunatsregierung also in ein Abhängigkeitsverhältnis gezwungen, das ihnen andererseits ihren sozialen Status sicherte. Mit den Worten von Bourdieu gesprochen ließe sich sagen, dass hier ökonomisches gegen soziales Kapital eingetauscht wurde. Wird bedacht, dass sich der Status eines bushi nach dessen Einkommen richtete, ${ }^{83}$ bedeutete dies zugleich einen festen Platz innerhalb der Samurai-Hierarchie einzunehmen. Besonders die niederen Samurai, bei denen das Rentenlehen vorherrschte, ${ }^{84}$ gerieten in völlige Abhängigkeit zu ihren Soldzahlungen. Insgesamt war es so zwar nicht mehr möglich seinen sozialen Status zu verlieren - ein Aufstieg schien jedoch ebenso unwahrscheinlich.

Diese durch das Shogunat gesetzten Kontrollmaßnahmen hatten auf Dauer gesehen einen buchstäblich hohen Preis. Da die Samurai aufgrund ihres verpflichtenden Aufenthalts in den Burgstädten ihre Ländereien nicht mehr selbst bestellen durften, waren sie aus ökonomischer Sicht unproduktiv. Der Unterhalt, welchen der shōgun und die Fürsten für ihr Gefolge aufbringen mussten, betrug durchschnittlich mehr als zwei Drittel des bei den Bauern eingetriebenen Steueraufkommens, was das Feudalsystem der Tokugawa langfristig auszehrte. ${ }^{85}$ Als Folge trat im 18. Jahrhundert ein Verarmungsprozess ein, der alle Herrschaftsebenen der Kriegeraristokratie umfasste. Die einzelnen Fürstentümer wurden neben den aufzubringenden Unterhaltskosten für ihr Samuraigefolge vor allem durch das System der wechselnden Residenzen finanziell erheblich belastet. Weitere Faktoren wie die Reduktion ihrer Jahreseinkommen durch die Regierung in Edo, dem gleichzeitigen Ansteigen der Abgaben für öffentliche Aufgaben oder zusätzliche Ausgaben wie etwa zur Bekämpfung von Hunger- oder Naturkatastrophen führten zwangsläufig zu einer Verschuldung bei den Kaufmannsfamilien. ${ }^{86}$ Zusätzlich verschärfte das Aufkommen der Geldwirtschaft die Gesamtsituation. Die Samurai, welche mittels Reis-Pensionen entlohnt wurden, waren dadurch gezwungen bei Kaufleuten Darlehen aufzunehmen, was die ohnehin bestehende Abhängigkeit zu diesen noch weiter verstärkte. ${ }^{87}$ Umgekehrt versuchten aber auch Teile der Händlerschaft sich unter Aufwendung ihres ökonomischen Kapitals in den höheren Stand einzukaufen, was durch die Adoption in Samurai-Familien möglich war. ${ }^{88}$

Abseits dieser ökonomischen Umbrüche brachten die Ordnungsmaßnahmen der Tokugawa-Herrschaft eine über zweihundert Jahre andauernde Friedensperiode mit sich, die eine Demilitarisierung und gleichzeitige Bürokratisierung des Samuraistandes zur Folge hatte. Hintergründig hierfür war wiederum das Bestreben des Shogunats den, sich im japanischen Mittelalter herausentwickelten, Militärstand zu domestizieren und die Kriegsführung zwischen den Samurai zu unterbinden. Von den Motiven

Geschichte Japans, S. 59.

83 Während die daimyō jährlich über mehr als 10.000 koku Reis verfügten, belief sich dieser Betrag bei den mittleren Samurai auf 500 bis 10.000 koku und bei den Fußsoldaten auf 15 koku: Schwentker, Die Samurai, S. 45. den von fürstlicher Hand eingezogenen und verwalteten Tributleistungen: Zöllner, Geschichte Japans, S. 42. 
her kann die Vorgangsweise des shōgun durchaus mit den Landfriedensbestrebungen der römisch-deutschen Könige verglichen werden. Ziel war, die im Kampf erworbenen Fähigkeiten der Samurai in einen Kodex der persönlichen Disziplin und Selbstdarstellung umzuwandeln. ${ }^{89}$ Der Übergang vom Krieger zum Bürokraten widersprach dem Selbstverständnis der Samurai also nicht grundlegend, da beide Positionen absolute Loyalität zu einem Herrn voraussetzten. ${ }^{90}$

Theoretischer Unterbau für diese Domestizierungsstrategien bot wiederum der Konfuzianismus, welcher die Berechtigung zum bürokratischen Dienst allein den bushi zugestand, denen damit statt der Kriegsführung eine neue Berufung zukam. Diese neuen Anforderungen führten im 17. und 18. Jahrhundert zu einer steigenden Anzahl von Samurai-Schulen sowie zur Entwicklung einer Gelehrtenschicht. ${ }^{91}$ Bis zum Ende der Tokugawa-Ära verfügten fast alle fürstlichen Herrschaftsgebiete über solche Schulen, die von den Kindern der Kriegerfamilien besucht wurden. Neben allgemeiner Bildung lag der Fokus auch hier auf dem militärischen Bereich. Finanziert und verwaltet wurden die Ausbildungsstätten durch die einzelnen Fürstentümer. ${ }^{92}$ Der dadurch gute Ausbildungsstand, den die Samurai im Vergleich zu anderen gesellschaftlichen Gruppen besaßen, qualifizierte sie auch in der Praxis für Positionen in der öffentlichen Verwaltung. Höhergestellte Samurai konnten sich als Zeremonienmeister oder politische Berater des shōgun in Edo betätigen, während andere in verschiedensten Behörden und Rechtsinstituten, von den Gerichten bis hin zum Strafvollzug, in der Aufsicht der buddhistischen Tempel und Shintō-Schreine, im Finanz- und Steuerwesen oder in der Verwaltung der Burgen, Städte und öffentlichen Verkehrswegen arbeiteten. Vor allem die mittleren Samurai-Ränge trugen zur Aufrechterhaltung der öffentlichen Verwaltung bei. Die meisten der ausgeübten Ämter wurden vererbt, während andere zumindest einen höheren Rang erforderten..$^{93}$

"[T]hey ruled by the virtue of their superior political and social status and through outright control of land, but at the same time, as individuals they largely lacked any socioeconomic base of independence." ${ }^{\prime 94}$

erklärte Douglas R. Howland. Es fasst die zwiespältige Situation der Samurai in der Tokugawa-Periode treffend zusammen: Zwar garantierte das ständisch-konfuzianische System ihren sozialen Rang, jedoch waren die Samurai bezüglich ihrer Kapitalformen in ein Abhängigkeitsverhältnis zum Shogunat geraten. Am deutlichsten zeigte sich dies in Bezug auf ihr ökonomisches und soziales Kapital, welches wie eingefroren erscheint. Betreffend ihres kulturellen Kapitals entfaltete sich hingegen in den Demilitarisierungsund Bürokratisierungsprozessen eine neue Dynamik und führte zu einer Neupositionierung - vom Krieger zum Beamten - innerhalb des Staatsapparats.

89 Howland, Samurai Status, S. 368.

90 Hirehido Sonoda, The Decline of the Japanese Warrior Class, 1840-1880, in: Japan Review 1 (1990), S. 73-111, hier S. 81.

91 Howland, Samurai Status, S. 371.

92 Sukanya Nitungkorn, Education and Economic Development during the Modernization Period. A Comparison between Thailand and Japan, in: Southeast Asian Studies 38 (2000), Heft 2, S. 142-164, hier S. 147.

93 Schwentker, Die Samurai, S. 47

94 Howland, Samurai Status, S. 368. 


\section{Einflüsse der Meiji-Restauration auf den Stand der Samurai}

\subsection{Vom Niedergang des Tokugawa-Shogunats zur Meiji-Restauration}

Ein weiteres, bisher unerwähntes Element der Tokugawa-Herrschaft stellte die fast völlige Abschottung des Landes nach außen dar. Zur inneren Machtkonsolidierung des Shogunats wurde dabei als Erstes die portugiesische Bevölkerung im Jahr 1639 des Landes verwiesen sowie in Folge das Christentum verboten und katholische Geistliche verfolgt. Japaner*innen, die sich länger als fünf Jahre im Ausland aufgehalten hatten, durften nicht mehr zurückkehren. Der einzige europäische Handelspartner blieben die an der Missionierung uninteressierten Niederlande, genauer gesagt deren OstindienKompanie. ${ }^{95}$

Ende des 18. Jahrhunderts kam es zu neuerlichen Kontakten mit dem expandierenden Zarenreich sowie mit Großbritannien, das die Aufnahme von Handelsbeziehungen vorschlug, was die jeweiligen daimyō allerdings ablehnten. Spätestens seit dem Opiumkrieg 1842/43 in China fürchtete Japan den Einflussgewinn fremder Mächte sowie die Auswirkungen von aufgezwungenen, ungleichen Handelsbeziehungen. Das Shogunat ordnete daraufhin eine Steigerung der Rüstungsanstrengungen und die verstärkte Befestigung der Küsten an. Langfristig brachte dies die einzelnen Fürstentümer in eine autonomere Position, wobei sich zugleich aber ihre finanzielle Lage weiterhin verschärfte. Neben den bereits beschriebenen Verarmungsprozessen kam es 1836 nach einer Reihe von Missernten zusätzlich zu Hungerrevolten. ${ }^{96}$

Die sozioökonomischen Missstände im Inneren führten - in Kombination mit dem Bedrohungspotential der imperialen Mächte seit den 1820er-Jahren - zum Aufschwung der sogenannten "Nationalen Schule" (kokugaku). Deren Wegbereiter strebten einen Zustand der inneren geistigen und politischen Geschlossenheit Japans an, um den Krisenerscheinungen der konfuzianisch geprägten Tokugawa-Staatsordnung entgegentreten zu können. Diesen Idealzustand sahen die frühen Anhänger der kokugaku im altjapanischen Staat respektive in der Person des tennō, der als oberster Priester und höchster politischer Souverän die Harmonie im Land wiederherstellen und nationale Einheit stiften sollte. ${ }^{97}$ Von dieser Situation ausgehend bildete die erzwungene Öffnung Japans 1853 ein entscheidendes Ereignis, das in den folgenden Jahrzehnten Dynamiken freisetzen sollte, die in die Entstehung des modernen japanischen Staates mündeten.

Unter dem Kommando von Matthew Calbraith Perry erreichten vier Schiffe der USKriegsmarine im Juli 1853 die Bucht von Edo. Den Beamten des shōgun wurde ein Brief des (zu dieser Zeit bereits nicht mehr amtierenden) Präsidenten Millard Fillmore, in dem er die Aufnahme von Handelsbeziehungen vorschlug, übergeben. Der Kaiser Fillmore meinte damit den shōgun - könne versuchsweise wagen, die bestehenden

95 Josef Kreiner, Japan und die ostasiatische Staatenwelt an der Wende vom Mittelalter zur Frühen Neuzeit, in: Josef Kreiner (Hrsg.), Geschichte Japans, Stuttgart 20186, S. 149-203, hier S. 200-201.

96 Krebs, Das moderne Japan, S. 3.

97 Wachutka, Der kokutai-Diskurs, S. 73-75. 
Gesetze für einige Jahre auszusetzen, um sich von den positiven Auswirkungen des Freihandels zu überzeugen. Mit der Ankündigung im kommenden Jahr zurückzukehren, um eine Antwort auf den Brief entgegenzunehmen, reiste Perry ab. ${ }^{98}$

Die Verhandlungsposition der Regierung in Edo erwies sich zu dieser Zeit als durchaus ungünstig, da noch im selben Monat der amtierende shōgun verstarb und sein Nachfolger erst im Dezember ernannt wurde. Das bakufu holte in der Zwischenzeit Stellungnahmen der Fürstentümer zu den Ereignissen ein. Deren Antworten spiegelten die brennenden Themen der Zeit wider: Die Gegner der Landesöffnung argumentierten, dass es durch den einsetzenden Handel zum Abfluss essentieller Waren kommen würde, was eine Teuerungswelle zur Folge hätte. Einzige Nutznießer einer solchen Entwicklung wären daher die Kaufleute. Einige andere daimyō sprachen sich hingegen für eine weitere Verbesserung der Landesverteidigung aus, forderten aber im Gegenzug eine Lockerung des verhassten Systems der alternierenden Residenzpflicht, um eine Aufrüstung finanzieren zu können. Diesen Vorschlag lehnte die Regierung jedoch ab. ${ }^{99}$ Aus dieser Position der Schwäche heraus akzeptierte die Shogunatsregierung bei der Wiederkehr Perrys schließlich die Bedingungen der USA, wodurch es am 31. März 1854 zum Abschluss des Vertrags von Kanagawa kam. In diesem verpflichtete sich die japanische Seite zu zahlreichen, für sie nachteiligen Bedingungen, wie etwa der Aufgabe ihrer Zollautonomie oder der Einrichtung von Konsulargerichten, die statt den japanischen Gerichten Prozesse gegen ausländische Staatsbürger*innen durchführten. In den kommenden Jahren schlossen immer mehr europäische Mächte solche „ungleichen Verträge" ab; zuletzt Österreich-Ungarn im Jahr 1869.100

Die lange im Raum stehenden Zweifel an der bestehenden Ordnung intensivierten sich nun mit den wachsenden Forderungen der ausländischen Mächte. Oppositionelle Kräfte gewannen an Zuwachs. In den südwestlichen Domänen Chōshū und Satsuma hatten einige Feudalherren mit der Einleitung militärischer Reformen begonnen und traten offen in Opposition zum bakufu. ${ }^{101}$ Auch der Kaiser widersetzte sich erstmals seit dem Beginn der Tokugawa-Herrschaft dem shōgun, indem er seine Zustimmung zu den Handelsverträgen verweigerte. Ursprünglich hatte die Regierung in Edo geplant ihre Handlungen durch den Segen des Kaiserhauses zu legitimieren, was allerdings fehlschlug. Stattdessen scharrte sich eine Oppositionsbewegung um den Kaiserhof.102

Bezüglich der Formierung dieser regierungsfeindlichen Bewegung kann wieder auf Bourdieu zurückgegriffen werden, da ihr Kern vor allem aus den „äußeren daimyō" bestand. Wie in dem Abschnitt zur Etablierung des Shogunats erklärt wurde, belehnte Tokugawa leyasu seine Verwandten und Verbündeten mit den Regionen um Edo. Sie besaßen also ein höheres soziales Kapital als die an die Peripherie verbannten

\footnotetext{
98 Zöllner, Geschichte Japans, S. 140-141.

99 Ebd., S. 141.

100 Christian Oberländer, Von den Ungleichen Verträgen zur Großmacht - Japans Weg zum modernen Nationalstaat, in: Josef Kreiner (Hrsg.), Geschichte Japans, Stuttgart 20186, S. 261-331, hier S. 265-266.

101 Frank Jacob, Die Meiji-Restauration und die Neuordnung Japans. Umverteilung und sozialer Wandel, in: Traverse 22 (2015), Heft 1, S. 79-92, hier S. 81.

102 Krebs, Das moderne Japan, S. 5.
} 
„äußeren daimyō. Diese sahen nun die Chance ihren Kapitalmissstand der vergangenen zweihundert Jahre auszugleichen, indem sie Beziehungen zu dem erstarkenden Kaiserhof knüpften. Die Einheirat des erst sechszehnjährigen shōgun in die kaiserliche Familie $1862^{103}$ demonstrierte diesbezüglich, dass die Tokugawa auch selbst begriffen hatten, wie schlecht es um ihre soziale Stellung stand. Spätestens hier wurde die Rolle des Kaiserhauses zur Generierung von sozialem Kapital eine unleugbare Tatsache.

Parallel zur weiteren Erosion der Macht des shögun schlossen sich nach und nach auch „innere daimyō" sowie Verwandte der Tokugawa der oppositionellen Bewegung an, die in Folge immer weniger Kompromissbereitschaft aufzeigte. Sie brachten im Januar 1868 den jungen tennō Mutsuhito in ihre Gewalt und verkündeten in seinem Namen die Restauration der Monarchie. Die daraufhin ausbrechenden militärischen Auseinandersetzungen zwischen den Streitkräften des Shogunats und der kaiserlichen Armee konnte Letztere schließlich für sich entscheiden. Als Motto seiner Regierungszeit wählte der Kaiser meiji, was übersetzt „erleuchtete Regierung" bedeutet. ${ }^{104}$

\subsection{Der Einfluss der Meiji-Restauration auf den Samuraistand}

Ein des Öfteren in der Fachliteratur diskutierter Punkt in Bezug auf die Meiji-Restauration stellt die scheinbare Paradoxie des Handelns der Samurai dar. So schreibt beispielsweise Frank Jacob in seinem Aufsatz, dass die daimyō mit dem Sturz des shōgun zu einem guten Teil gegen die Interessen ihres eigenen Standes arbeiteten.

„Denn selbst wenn ihr politischer Einfluss, ihre wirtschaftliche Potenz sowie ihr sozialer Status in den letzten Dekaden der Tokugawa-Herrschaft gesunken waren, waren die meisten Samurai nicht gewillt, die bestehende Ordnung als solche abzuschaffen und durch eine neue zu ersetzen."105

In Bezug auf die formell bestehenden Vorrechte der bushi scheint diese Aussage zunächst zuzutreffen. Allerdings bieten die bisher dargelegten Ausführungen gute Argumente für die These, dass ein Großteil der Samurai den für sie vorgesehenen Rang in der Gesellschaft nicht mehr einnehmen konnte. Dazu reichte die Gesamtheit ihres Kapitals - vor allem des ökonomischen - schlicht nicht mehr aus. Die im vorigen Abschnitt geschilderten, prekären Zustände verdeutlichten dies besonders. Was die Situation der Samurai zur Mitte des 19. Jahrhunderts betrifft, nennt Wolfgang Schwentker zahlreiche Quellenbeispiele, die die soziale Wirkung ihrer Verarmung aufzeigen. Beispielsweise verbeugten sich, was im 17. Jahrhundert noch unvorstellbar gewesen wäre, Samurai vor Kaufleuten und ihnen wurden Darlehen von Vertretern der restlichen Stände nicht gewährt. Andere gaben wiederum ihre Privilegien auf, um an den Vorteilen einer Tätigkeit als Handwerker oder Händler teilzuhaben. Etwaige Reformversuche in der späten Tokugawa-Zeit blieben weitgehend wirkungslos. ${ }^{106}$

\footnotetext{
103 Krebs, Das moderne Japan, S. 6.

104 Ebd.

105 Jacob, Die Meiji-Restauration, S. 81

106 Schwentker, Die Samurai, S. 51-52.
} 
Mit der Machtübernahme des Kaiserhofes kam es zur schrittweisen Neuordnung des sozialen Raumes. 1869 löste die neue Regierung die bisherige Ständeordnung auf und schuf das Feudalsystem mit der Aufhebung der fürstlichen Domänen formal ab. Ziel war es nun eine direkte Verbindung zwischen Staat und Individuum herzustellen. ${ }^{107}$ Die daimyō wurden nach der Rückgabe ihrer Lehen an den Kaiser zu den Gouverneuren der jeweiligen Provinzen bestimmt, aber ab 1871 von kaiserlichen Beamten abgelöst. Nach einer völligen Neuordnung des Landes waren die über 260 Fürstentümer zu 45 Präfekturen zusammengelegt worden, deren Leitungsbeamte ihre Anweisungen direkt von der Zentralregierung erhielten. Die ehemaligen Fürsten und ihre Anhänger leisteten dagegen keinen Widerstand, da die neue Zentralregierung sämtliche ihrer Schulden annullierte oder übernahm. Zudem wurden sie persönlich sehr großzügig abgefunden. Durch die Erfahrungen der letzten Jahrzehnte kamen viele von ihnen auch zu dem Schluss, dass sich Japan ohne einen starken Einheitsstaat nicht gegen die westlichen Großmächte behaupten können würde, weshalb sie diese Umstrukturierungen billigten. ${ }^{108}$

Trotz der Abschaffung der feudalen Strukturen kann von einer vollständigen Beseitigung der sozialen Unterschiede jedoch nicht die Rede sein. Vielmehr wurde das bestehende Ständesystem in eine Klassengesellschaft transformiert. An deren Spitze stand der Hochadel (kazoku), der sich aus den ehemaligen daimyō sowie dem Hofadel zusammensetzte. Der ehemalige Samuraistand bildete die Klasse der Kriegerfamilien oder shikozu, während alle anderen Bewohner*innen des neu entstandenen Kaiserreiches den "Gemeinen" (heimin) zugeordnet wurden. ${ }^{109}$ Einhergehend mit dieser Neueinteilung kam es zu zahlreichen Maßnahmen, welche die soziale Mobilität der einzelnen Klassen wesentlich förderte. So erhielten 1870 alle Bürger*innen das Recht einen Familiennamen zu tragen und die Eheschließung wurde nicht mehr durch ständische Grenzen eingeschränkt. Auch die Sonderstände der sozial ausgeschlossenen Gruppierungen ${ }^{110}$ wurden abgeschafft. Den Samurai war es nun problemlos möglich ihren Beruf frei zu wählen. ${ }^{111}$ Allerdings relativierte die Einebnung dieser ständischen Unterschiede auch die Rolle der ehemaligen bushi, indem ihre Reis-Stipendien abgelöst und verschiedene Vorrechte, wie Angehörige aus dem Volk körperlich zu züchtigen oder zwei Schwerter tragen zu dürfen, annulliert wurden. ${ }^{112}$

Gerade die letzten Punkte missfielen aber auch einigen Angehörigen der Kriegerfamilien, die sich in ihrem Selbstverständnis erschüttert sahen. Die eingeleiteten Maßnahmen der Meiji-Regierung boten zwar mehr soziale Mobilität für alle, doch bedeuteten sie für eine Minderheit auch zwangsläufig die Aufgabe von althergebrachten Privilegien und Gewohnheiten. Als weiterer Bruch wird hierbei auch die Einführung der allgemeinen Wehrpflicht genannt, ${ }^{113}$ durch welche der ehemaligen militärischen Elite nun

107 Walker, A Concise History, S. 161.

108 Zöllner, Geschichte Japans, S. 196-197.

109 Schwentker, Die Samurai, S. 60.

110 Siehe Fußnote 45.

111 Zöllner, Geschichte Japans, S. 198.

112 Schwentker, Diener und Herrscher, S. 28-29.

113 Schwentker, Samurai und Soldaten, S. 139-141; Jacob, Die Meiji-Restauration, S. 87; Zöllner, Geschichte Japans, S. 210-211. 
auch formal ihre ursprünglichste Existenzberechtigung entzogen wurde. Die Tatsache, dass sich Vertreter dieses traditionalistischen Verständnisses nach der Abschaffung der letzten Samurai-Privilegien 1877 in der Satsuma-Rebellion gegen die Zentralregierung erhoben, führte zu einer regen Forschungsdiskussion darüber, ob die ehemaligen Samurai nun zu den Gewinnern oder Verlierern der Restauration gehörten. Bei der genaueren Betrachtung dieser Erhebungen, die letztendlich scheiterten, zeigt sich jedoch, dass die Aufständischen verschiedene politische Ziele verfolgten und quantitativ nur rund sechs Prozent der Samurai an ihnen teilnahmen. ${ }^{114}$ Insgesamt stellten sie eher eine Ausnahmereaktion als die allgemeine Antwort des Samuraistandes auf dessen Auflösung dar. ${ }^{115}$ Daraus ergibt sich wiederum die Frage, auf welche Kapitalformen diejenigen ehemaligen Samurai, die sich nicht an den Aufständen beteiligten, zurückgreifen konnten.

Gerade die Umstrukturierung des Militärs bot vielen Angehörigen der einstigen Kriegerklasse eine Perspektive - jedoch nicht als Soldaten, sondern als Bürokraten. Bezüglich ihres Anteils im Verwaltungspersonal der militärischen Dienststellen in den späten 1870er-Jahren nennt Wolfgang Schwenkter für das Marine- und Heeresamt Zahlen von 75 respektive 85 Prozent. ${ }^{116}$ Bei der Betrachtung der gesamten öffentlichen Verwaltung zeigt sich, dass im Jahr 1873 fast achtzig Prozent der Beamtenschaft in den Institutionen Tokios und den Präfekturen der shikozu-Klasse angehörten. Diese Monopolstellung im administrativen Sektor setzte sich in den folgenden Jahrzehnten fort, wie die Studierendenzahlen an der Universität von Tokio zeigen, in welcher die künftigen Verwaltungskräfte ausgebildet wurden. Dort stammten 1883 noch sieben von zehn Studierenden aus Samurai-Familien. ${ }^{117}$ Schlüsselpositionen innerhalb des Verwaltungsapparats konnten bis zur Einführung von Staatsprüfungen 1887 nur durch Ernennungen besetzt werden. So ernannte beispielsweise der Kaiser die Beamten, welche in der Zentralregierung zum Einsatz kamen. ${ }^{118}$ Diese Spitzenfunktionäre stammten meist aus Chōshū und Satsuma, ${ }^{119}$ also denjenigen Provinzen, die sich als Erstes gegen den shōgun gewandt hatten. Hier spielte also nicht nur das Bildungsniveau der Kandidaten eine Rolle, sondern auch ihre Vernetzung mit dem Kaiserhof.

Abseits der bürokratischen Strukturen konnten die shikozu mit dem allmählichen Entstehen eines Parteiensystems in Japan ${ }^{120}$ auch vermehrt im politischen Feld aktiv werden. Dabei knüpften sie an ihre bisherige Führungsrolle als lokale Eliten in den Fürstentümern an. Durch die gesellschaftliche Pluralität, die die Meiji-Restauration mit sich brachte, engagierten sich neben den ehemaligen Samurai aber auch wohlhabende ländliche Grundbesitzer, Unternehmer sowie Geschäftsleute in der Politik. Diese konsolidierten sich teilweise viel stärker in bestimmten Parteien, während die shikozu in

\footnotetext{
114 Schwentker, Die Samurai, S. 66

115 Ursula Koike-Good, Die Auflösung der Samuraiklasse und die Samuraiaufstände. Ein Beitrag zur japanischen Geschichte von 1868 bis 1878 (Schweizer asiatische Studien 16), Bern 1994, S. 7.

116 Schwentker, Samurai und Soldaten, S. 143.

117 Schwentker, Die Samurai, S. 66-67.

118 Zöllner, Geschichte Japans, S. 264.

119 Ebd.

120 Krebs, Das moderne Japan, S. 12-13
} 
allen politischen Lagern vertreten waren. ${ }^{121}$ Am Rande sei hier noch erwähnt, dass der erste Premierminister des Kaiserreiches, Itō Hirobumi, ebenfalls der Sohn eines Samurai war. Allerdings stammte er nicht aus einer alteingesessenen Kriegerfamilie, sondern bekleidete einen der niedrigsten Ränge in der Samurai-Hierarchie. In seiner Position als Leiter der Regierung besaß er aber bedeutend mehr Einfluss als die ehemaligen daimyō. ${ }^{122}$ Als Teil des Hochadels vertraten diese ihre politischen Interessen im 1890 geschaffenen Oberhaus des Parlaments. ${ }^{123}$ Itō, der sowohl seine Ausbildung und Herkunft als Samurai als auch die soziale Mobilität der Meiji-Gesellschaft für sich nutzte, vereinte sprichwörtlich das Beste aus beiden Welten.

Da aber natürlich nicht alle Mitglieder der vormaligen Kriegeraristokratie in Positionen der Verwaltung und Politik untergebracht werden konnten, startete die Regierung in Tokio ein umfassendes Hilfsprogramm. ${ }^{124}$ Ziel dabei war es, die Samurai, welche den Staatshaushalt wesentlich belasteten, ökonomisch unabhängig zu machen. Denn mit der Auflassung der Fürstentümer 1871 übernahm die Zentralregierung auch die Verantwortung für deren Besoldung, die rund ein Drittel der gesamten Staatsausgaben ausmachte. Zunächst versuchte die Regierung Anreize zur Ergreifung eines bürgerlichen Berufes zu schaffen, wofür den ehemaligen Samurai im Gegenzug eine Abfindung in der Höhe von mehreren Jahresgehältern in Aussicht gestellt wurde. Knapp 230.000 Bezugsberechtigte ließen sich darauf ein; die restlichen Samurei ereilte eine Reihe von Soldkürzungen, bis diese 1876 völlig eingestellt und in niedrig verzinste Staatsanleihen umgewandelt wurden. ${ }^{125}$ Der Verlust dieser Unterhaltsansprüche trug neben der bereits erwähnten Abschaffung anderer Privilegien wesentlich zur im Folgejahr ausbrechenden Satsuma-Rebellion bei. ${ }^{126}$

Die nun eingeleiteten Hilfsmaßnahmen sollten einerseits den Samurai neue Beschäftigungsmöglichkeiten bieten, aber auch zur Modernisierung des Landes beitragen. Im Vordergrund stand dabei vor allem das Projekt der Kolonisierung der nördlichsten Hauptinsel Japans, Hokkaido, sowie anderer peripherer Regionen. Insgesamt beteiligten sich zwanzigtausend Samurai mit ihren Familien an den Vorhaben zur LanderschlieBung, wobei sie ihre Anleihescheine in Papiergeld umtauschen und zu günstigen Konditionen Ackerland pachten konnten. Allein auf Hokkaido, welches aufgrund russischer Übergriffe auch strategische Bedeutung besaß, beteiligten sich zwischen 1884 und 1889 rund fünftausend Samurai. Als zweiten Weg, um die erhaltenen Staatsanleihen in Geld umtauschen zu können, wurde eine Beteiligung an der neu gegründeten Nationalbank vorausgesetzt. Hintergedanke der Regierung beim Ausbau der Filialstruktur war eine Belebung des Warenhandels. Am Ende der 1870er-Jahre kam es daher zum Aufbau von knapp 150 Zweigstellen der Nationalbank, die allesamt ehemalige Samurai leiteten. Außerdem wurden ihnen günstige Darlehen angeboten, um in neue Indust-

121 Oberländer, Von den Ungleichen Verträgen, S. 309.

122 Shinichi Kitaoka, The Significance of the Meiji Restoration, in: Asia-Pacific Review 25 (2018), Heft 1, S. 5-18, hier S. 14 .

123 Schwentker, Diener und Herrscher, S. 29.

124 Ebd., S. 29

125 Zöllner, Geschichte Japans, S. 211.

126 Oberländer, Von den Ungleichen Verträgen, S. 296-297. 
rieprojekte - vor allem in der Bauwirtschaft - zu investieren. All diese Aspekte führten in den 1970er- und 80er-Jahren zu diversen Publikationen, die den wirtschaftlichen Aufstieg Japans zur zweitgrößten Industrienation der Welt durch die Unternehmertätigkeit der ehemaligen Samurai zu rechtfertigen versuchten, wobei allerdings die zahlreichen Fehlschläge von „Samurai-Unternehmern“ nicht berücksichtigt wurden. ${ }^{127}$

\section{Fazit}

Durch den gezeigten Längsschnitt ergibt sich eine mehrfache Umverteilung des sozialen, kulturellen und ökonomischen Kapitals der Samurai, die sich vor, während und vor allem nach der Herrschaft der Tokugawa zutrug. In Summe lassen sich aus den dargelegten Ausführungen zwei Phasen mit drei Brüchen herausarbeiten.

Zum Ersten wäre die Reichseinigung unter den Tokugawa zu nennen, welche langfristig eine Domestizierung des Samuraistandes zur Folge hatte. Das inkorporierte kulturelle Kapital, über welches seine Angehörigen verfügten, verschob sich dabei von militärischen Fähigkeiten wie der Waffenhandhabung und des strategischen Denkens hin zu einer allgemeineren Bildung, die sie für administrative Positionen qualifizierte. In beiden Fällen handelte es sich um kulturelles Kapital, das zur Herrschaftsausübung benötigt wurde, doch aufgrund der friedlicheren politischen Rahmenbedingungen schwand die praktische Notwendigkeit der Samurai als Krieger zunehmend. Trotz dieses Funktionswechsels behielten die bushi aber weiterhin standesübliche Privilegien, die ihnen jedoch nicht immer zum Vorteil gereichten. So unterbanden die herrschaftssichernden Einrichtungen des Shogunats ein Anwachsen des sozialen und vor allem des ökonomischen Kapitals der Samurai. Vielmehr befanden sich diese beiden Bereiche in einem statischen Zustand - das ökonomische Kapital schwand sogar. Die erste Phase, die die Edo-Zeit umfasst, wäre daher als "Gefangenschaft in Privilegien" zu betiteln.

Die zweite Phase, die mit der Meiji-Restauration anbrach, ist dementsprechend als Befreiung zu deuten, die allerdings auch ihre Risiken barg. Während vor allem die daimyō mit dem Wegfall der wechselnden Residenzpflicht sowie der Besoldung ihrer Anhänger erheblich an ökonomischen Kapital gewinnen konnten, trat in den unteren Samuraischichten zunächst keine Besserung der bestehenden Verhältnisse ein. Was das soziale Kapital der vormaligen Kriegeraristokratie betrifft, lässt sich insgesamt ein Wechsel der Vorzeichen beobachten. Diejenigen Samuraifürsten, welche ein vertrautes Verhältnis zum shōgun pflegten oder mit den Tokugawa verwandt waren, wurden dafür entmachtet, während vor allem die „äußeren daimyō“ ihre Beziehungsnetzwerke zum Kaiserhof nutzten, um erheblichen Einfluss in der Gestaltung des modernen Japan zu gewinnen. Mit der Etablierung des Parteiensystems hatten aber auch die weniger bevorteilten Samurai die Gelegenheit ihr soziales Kapital zu steigern. 
Die Frage, ob die Samurai ihr Kapital, also ihre gesellschaftliche Stellung, verspielten, lässt sich in Summe verneinen. Vor allem ihr inkorporiertes kulturelles Kapital, welches sie bereits in der Tokugawa-Ära erworben hatten, bildete die Grundlage, um ihre soziale Stellung trotz Annullierung der Standesunterschiede aufrechterhalten zu können. Wie geschildert erlangten auch jene, die diese Kontinuität nicht für sich nutzen konnten, die Gelegenheit ihr ökonomisches Kapital und damit ihre Position in der MeijiGesellschaft zu steigern. Damit konnten zuletzt auch die Samurai, welche zunächst von der Dynamik der Meiji-Restauration ausgeschlossen waren und sich vorrangig auf ihren symbolischen Privilegienverlust fixierten, letztendlich in den 1869 einsetzenden Modernisierungsprozess integriert werden. Die Chancen der shikozu-Klasse, zumindest ihr soziales und ökonomisches Kapital zu vermehren, waren im Gegensatz zur Tokugawa-Herrschaft wesentlich größer. Allerdings ging sie mit dem Verlust der bis dahin bestehenden Standessicherheit einher, da die neue Dreiklassengesellschaft bessere Möglichkeiten bot, die „feinen Unterschiede" auszugleichen.

\section{Literatur}

Asch, Ronald G., "The Politics of Access". Hofstruktur und Herrschaft in England unter den frühen Stuarts, 1603-1642, in: Werner Paravicini (Hrsg.), Alltag bei Hofe. 3. Symposium der Residenzen-Kommission der Akademie der Wissenschaften in Göttingen, Ansbach, 28. Februar bis 1. März 1992 (Residenzenforschung 5), Sigmaringen 1995, S. $243-266$.

Bourdieu, Pierre, Die verborgenen Mechanismen der Macht (Schriften zu Politik \& Kultur 1), Hamburg 1992.

Ders., Praktische Vernunft. Zur Theorie des Handelns, Frankfurt am Main 1998.

Ders., Sozialer Raum, symbolischer Raum (1989), in: Susanne Hauser/Christa Kamleithner/ Roland Meyer (Hrsg.), Architekturwissen. Grundlagentexte aus den Kulturwissenschaften, Bd. 1, Bielefeld 2011, S. 304-313.

Ders./Wacquant, Loïc, Reflexive Anthropologie, Frankfurt am Main 1996.

Dartmann, Christoph/Füssel, Marian/Rüther, Stephanie (Hrsg.), Raum und Konflikt. Zur symbolischen Konstituierung gesellschaftlicher Ordnung in Mittelalter und Früher Neuzeit, Münster 2004.

Dimbath, Oliver, Einführung in die Soziologie, o. O. $2016^{3}$.

Distelrath, Günther, Die vorindustrielle Dynamik der Frühen Neuzeit, in: Josef Kreiner (Hrsg.), Geschichte Japans, Stuttgart 20186, S. 204-260.

Hall, John Whitney, Das japanische Kaiserreich (Fischer Weltgeschichte 20), Frankfurt am Main $2009^{15}$.

Howland, Douglas R., Samurai Status, Class, and Bureaucracy. A Historiographical Essay, in: The Journal of Asian Studies 60 (2001), Heft 2, S. 353-380. 
Jacob, Frank, Die Meiji-Restauration und die Neuordnung Japans. Umverteilung und sozialer Wandel, in: Traverse 22 (2015), Heft 1, S. 79-92.

Kitaoka, Shinichi, The Significance of the Meiji Restoration, in: Asia-Pacific Review 25 (2018), Heft 1, S. 5-18.

Koike-Good, Ursula, Die Auflösung der Samuraiklasse und die Samuraiaufstände. Ein Beitrag zur japanischen Geschichte von 1868 bis 1878 (Schweizer asiatische Studien 16), Bern 1994.

Krebs, Gerhard, Das moderne Japan 1868-1952. Von der Meiji-Restauration bis zum Friedensvertrag von San Francisco (Oldenbourg Grundriss der Geschichte 36), München 2009.

Kreiner, Josef (Hrsg.), Geschichte Japans, Stuttgart $2018^{6}$.

Ders., Japan und die ostasiatische Staatenwelt an der Wende vom Mittelalter zur Frühen Neuzeit, in: Josef Kreiner (Hrsg.), Geschichte Japans, Stuttgart 20186, S. 149-203.

Lubbadeh, Jens, Samurai-Schau. Das Geheimnis der todbringenden Ritter, in: Der Spiegel, 24.2.2008, https://www.spiegel.de/wissenschaft/mensch/samurai-schau-dasgeheimnis-der-todbringenden-ritter-a-536951.html, eingesehen 7.3.2020.

Mathias, Regine, Shogunat, in: Enzyklopädie der Neuzeit, http://dx.doi.org/ 10.1163/2352-0248_edn_COM_348940, eingesehen 2.2.2020.

Nitungkorn, Sukanya, Education and Economic Development during the Modernization Period. A Comparison between Thailand and Japan, in: Southeast Asian Studies 38 (2000), Heft 2, S. 142-164.

Oberländer, Christian, Von den Ungleichen Verträgen zur Großmacht - Japans Weg zum modernen Nationalstaat, in: Josef Kreiner (Hrsg.), Geschichte Japans, Stuttgart $2018^{6}$, S. 261-331.

Ryūichi, Nagao, Der Staat Japan in historischer Perspektive, in: Kazuhiro Takii/Michael Wachutka (Hrsg.), Staatsverständnis in Japan. Ideen und Wirklichkeiten des japanischen Staates in der Moderne (Staatsverständnisse 90), Baden-Baden 2016, S. 13-45.

Saaler, Sven, Die Bedeutung der Epochenmarke 1868 in der Japanischen Geschichte. Restauration, Revolution, Reform, in: Saeculum 56 (2005), Heft 1, S. 69-104.

Schulze, Winfried, Einführung in die Neuere Geschichte, Stuttgart $2010^{5}$.

Schwentker, Wolfgang, Die Samurai im Zeitalter der Meiji-Restauration. Elitenwandel und Modernisierung in Japan, 1830-1890, in: Geschichte und Gesellschaft 28 (2002), Heft 1, S. 33-70.

Ders., Samurai und Soldaten. Zum Wandel des Militärs im Japan der Restaurationszeit, 1853-1895, in: Thomas Kolnberger (Hrsg.), Krieg und Akkulturation (Expansion - Interaktion - Akkulturation 5), Wien 2004, S. 130-152. 
Ders., Diener und Herrscher. Die japanische Geschichte im Spiegel der Samurai, in: Historisches Museum der Pfalz Speyer (Hrsg.), Samurai. Begleitbuch zur Ausstellung „Samurai" im Historischen Museum der Pfalz Speyer, Ostfildern 2008, S. 15-29.

Sonoda, Hirehido, The Decline of the Japanese Warrior Class, 1840-1880, in: Japan Review 1 (1990), S. 73-111.

Spafford, David, Emperor and Shogun, Pope and King. The Development of Japan's Warrior Aristocracy, in: Bulletin of the Detroit Institute of Arts 88 (2014), Heft 1, S. 10-19.

Takii, Kazuhiro/Wachutka, Michael (Hrsg.), Staatsverständnis in Japan. Ideen und Wirklichkeiten des japanischen Staates in der Moderne (Staatsverständnisse 90), BadenBaden 2016.

Wachutka, Michael, Der kokutai-Diskurs im Staatsverständnis des 19. und frühen 20. Jahrhunderts. Kerngedanken und Entwicklung der Idee von Japans einzigartigem Nationalwesen, in: Kazuhiro Takii/Michael Wachutka (Hrsg.), Staatsverständnis in Japan. Ideen und Wirklichkeiten des japanischen Staates in der Moderne (Staatsverständnisse 90), Baden-Baden 2016, S. 65-88.

Walker, Brett L., A Concise History of Japan, Cambridge 2015.

Zöllner, Reinhard, Geschichte Japans. Von 1800 bis zur Gegenwart, Paderborn 2006.

Ders., Samurai und Ritter, in: Historisches Museum der Pfalz Speyer (Hrsg.), Samurai. Begleitbuch zur Ausstellung "Samurai" im Historischen Museum der Pfalz Speyer, Ostfildern 2008, S. 173-179.

Maximilian Gröber studiert Geschichte sowie Katholische Fachtheologie an der Universität Innsbruck. maximilian.groeber@student.uibk.ac.at

\section{Zitation dieses Beitrages}

Maximilian Gröber, Verspieltes Kapital? Der gesellschaftliche Rollenwandel des Samuraistandes im Zuge der Meiji-Restauration, in: historia.scribere 13 (2021), S. 71-96, [http://historia.scribere.at], eingesehen 22.6.2021 (=aktuelles Datum).

Creative Commons Licences 3.0 Österreich unter Wahrung der Urheberrechte der Autorlnnen. 